\title{
Estudio de microscopía óptica y de tomografía axial computerizada del ligamento anular del carpo en fetos
}

\author{
E. MackenneY ${ }^{(1)}$, E. ANDRAdA ${ }^{(2)}$, J. HernáNDEZ ${ }^{(3)}$ \\ (1) JeFE de Unidad de Referencia de Mano. Hospital General Universitario de ElChe \\ (2) MÉdico Especialista de ANatomía Patológica. Hospital General Universitario de ElChe \\ (3) Médico Residente. Servicio de Cirugía Ortopédica y Traumatología. Hospital General \\ UNIVERSITARIO DE ELCHE
}

Correspondencia:

Dr. Enrique MacKenney Carrasco

Apartado 48

03130 Santa Pola

Alicante

e-mail: emackenney@coma.es

\begin{abstract}
El propósito de la investigación ha sido el estudio de la anatomía microscópica y de tomografía axial computerizada del ligamento anular del carpo en fetos, para determinar las características del ligamento anular del carpo, definir sus inserciones radial y cubital y sus relaciones con el contenido del túnel del carpo. Se estudió el ligamento anular del carpo de diez fetos en su tercer trimestre, y se realizó una TAC en cuatro. Las muestras se tiñeron con Hematoxilina Eosina y con Masson tricrómico y se realizaron microfotografías. El análisis de la microscopía óptica y de los cortes de TAC permite obtener el contorno y configuración del túnel del carpo, evidenciando las variaciones que experimenta según su localización de proximal a distal; diferenciándose el ligamento carpi volare proximal, del ligamento anular anterior del carpo, más distal. En el borde radial del ligamento se observa un esquema estructural que constituye la inserción externa del ligamento anular del carpo, diferenciado en tres zonas, en una transición sin límites netos entre una y otra zona. Existe una relación de contigüidad y no de continuidad, entre el ligamento anular del carpo y las capas más superficiales del epineuro del nervio mediano.
\end{abstract}

Palabras clave: túnel, carpo, ligamento anular, microscopía óptica
The objective of the research was to study the microscopic anatomy and the axial computerized tomographic morphology of the annular carpal ligament in fetuses in order to determine its characteristics, define its insertions and relationship with the content of the carpal tunnel. The annular carpal ligament was studied in ten fetuses. A computerized axial tomography was done in four. The samples were stained with hematoxiline-eosin and Masson-trichromic. Microphotographs of the annular carpal ligament were taken, from proximal to distal, assessing its structure and relationships. On computerized axial tomography, the outline and configuration of the carpal tunnel are defined, as well as the variations, according to the level from proximal to distal. On optical microscopy the anterior annular carpal ligament showed, in the central region, a structure of transversally oriented collagen fibres with long cells or fibroblasts. The radial edge of the ligament has a structural pattern consisting of the external insertion of the annular carpal ligament, differentiated in three zones and the transition from one zone to another being gradual with no defined limits. There is a relationship of contiguity and not of continuity between the annular carpal ligament and the synovium of flexor tendon and the perineurium.

Key Words: carpal tunnel, annular carpal ligament, optic microscopy. 


\section{INTRODUCCIÓN}

1 n las descripciones anatómicas del túnel del carpo, la parte ligamentosa forma la pared anterior o techo; distinguiéndose en ella una parte proximal o ligamento carpi volare, que es un engrosamiento de la fascia antebraquial $^{1}$, pero también descrita como parte superficial ${ }^{2}$ y el retináculo de los flexores o ligamento anular anterior del carpo ${ }^{1}$; o como distinguen Landsmeer ${ }^{3}$ y Kauer y Landsmeer ${ }^{4}$, una zona de transición entre antebrazo y la primera fila del carpo, una zona carpiana proximal, una zona carpiana distal y una zona de la base de los metacarpianos.

El interior del túnel del carpo está cubierto por una extensión de la fascia volar interósea, que recubre toda la superficie interna del túnel ${ }^{5}$.

El estudio histológico del retinaculum flexor ha mostrado la existencia de un gran número de fibras de colágeno orientadas transversalmente y de un grosor promedio de aproximadamente 10 veces el de la fascia antebraquial. En contraste las fibras de colágeno en la fascia antebraquial se orientaban longitudinalmente, permitiendo por lo tanto una diferenciación histológica entre el retinaculum flexor proximal y la fascia antebraquial $^{6}$.

El propósito de la investigación ha sido el estudio de la anatomía microscópica del ligamento

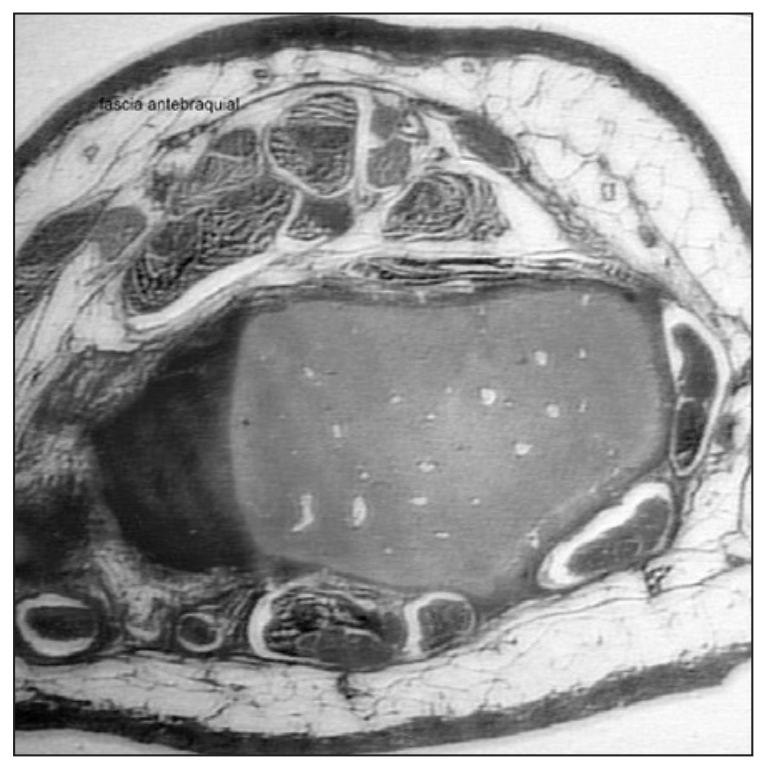

Figura 1. Microscopía óptica (MO). Túnel del carpo proximal. Fascia antebraquial.

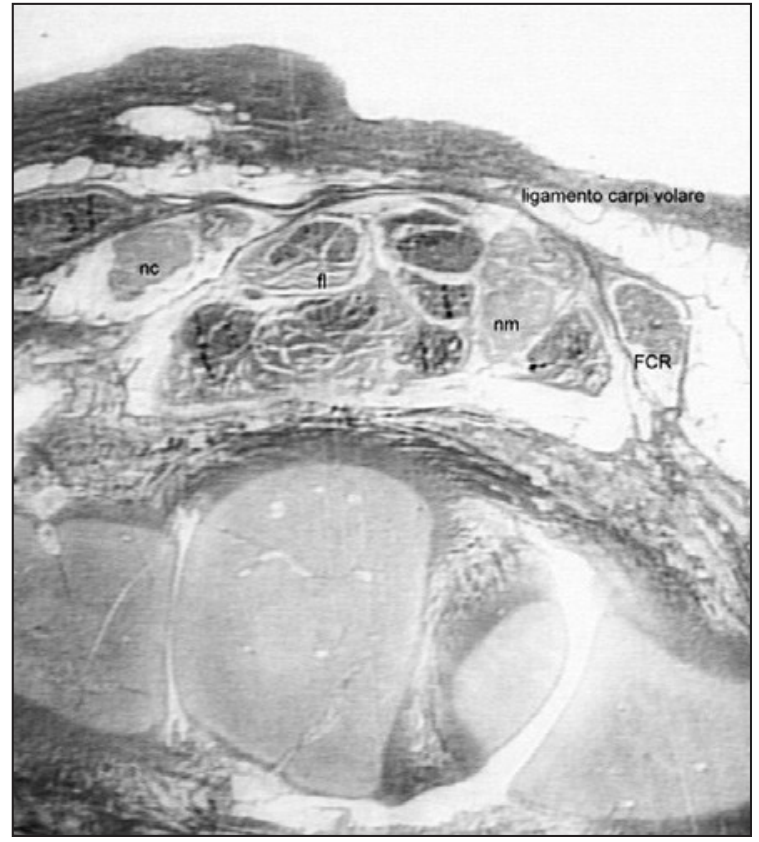

Figura 2. MO. Túnel del carpo zona proximal. Ligamento carpi volare.

anular del carpo en fetos, para determinar las características del ligamento anular del carpo, definir sus inserciones radial y cubital y sus relaciones con el contenido del túnel del carpo.

\section{MATERIAL Y MÉTODO}

Se estudió el ligamento anular del carpo de diez fetos en su tercer trimestre, disecados microquirúrgicamente con gafa-lupa $4 \mathrm{X}$, y utilizando la técnica de $\mathrm{McGrouther}^{7}$, que reseca la piel por pequeños segmentos, pero sólo después de una visualización de las inserciones de la piel a la fascia o tejido celular subyacente. En cuatro piezas se retiró quirúrgicamente el contenido del túnel del carpo y se realizó un estudio de tomografía axial computerizada previo a su procesado.

Las muestras fueron fijadas en formol y posteriormente procesadas mediante deshidratación e inclusión en bloques de parafina y talladas con microtomo. Se procedió a tinción con hematoxilina-eosina y tinción de Masson-Tricrómico.

Se realizaron microfotografías del ligamento anular del carpo a diferentes niveles, desde proximal a distal y desde radial a cubital, valorando su estructura y relaciones. 


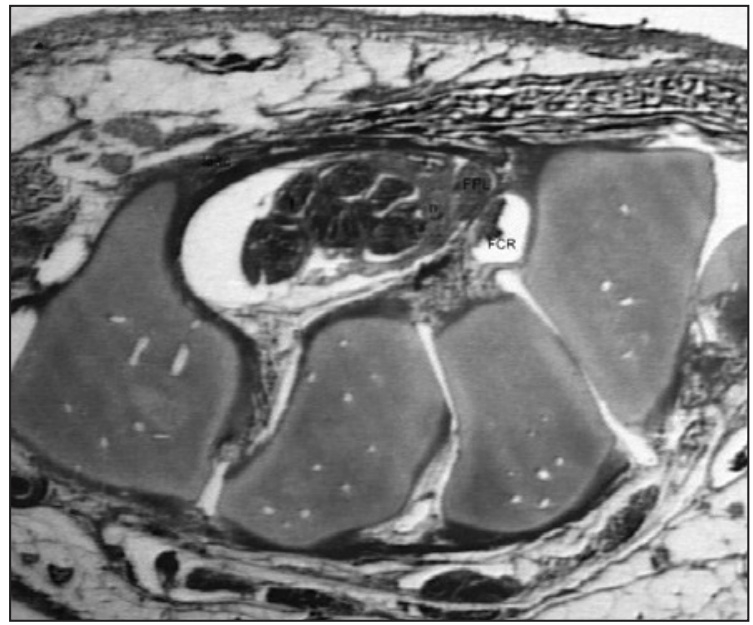

Figura 3. MO. Túnel del carpo distal. Ligamento anular del carpo.

\section{RESULTADOS}

En la disección de la región proximal de la palma de la mano en el feto se define perfectamente la estructura anatómica del túnel del carpo, distinguiéndose su extremo proximal en la articulación radio-carpiana, con un contorno ovalado, que disminuye progresivamente de altura de proximal a distal, a la vez que aumenta el grosor de la pared anterior, diferenciándose el ligamento carpi volare proximal, del ligamento anular anterior del carpo, mas distal (Figuras 1, 2 y 3).

El análisis de las muestras de microscopía óptica y de los cortes de TAC permite obtener el contorno y configuración del túnel del carpo, evidenciando las variaciones que experimenta según su localización de proximal a distal (Figuras 4 y 5).

El nervio mediano adopta dos posiciones en el túnel del carpo, radial o central. La capa más superficial de epineuro constituye una capa

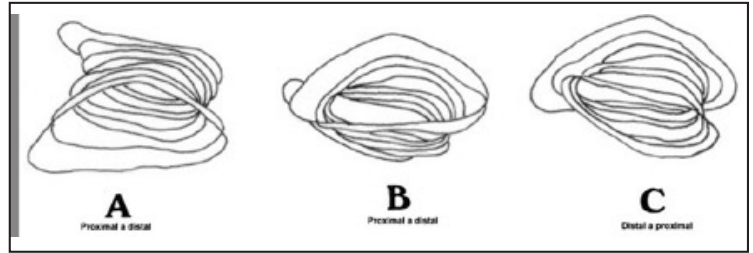

Figura 5. Contorno y configuración del túnel del carpo.

contigua al ligamento anular del carpo, pero con unas características histológicas que permiten su diferenciación del ligamento anular (Figura 6).

En su zona central y en el tercio cubital el ligamento anular toma relación con los tendones flexores de los dedos, en forma indirecta, a través de una capa de sinovial que rodea a todos y cada uno de los tendones.

En el borde radial del ligamento se observa un esquema estructural que constituye la inserción externa del ligamento anular del carpo, diferenciado en tres zonas (Figura 7), en una transición sin límites netos entre una y otra zona. Se distingue una zona I, que consiste en el ligamento propiamente tal, constituido por fibras de colágeno más o menos paralelas, con fibroblastos, que son las únicas células en esta zona. Una zona II consistente en fibrocartílago, con fibras de colágeno en una distribución irregular, con células ovaladas, mas parecidas a condrocitos. Y una zona III, que corresponde a tejido cartilaginoso organizado con células redondeadas. En esta zona se observa como las fibras de fibrocartílago adoptan una disposición perpendicular u oblicua al cartílago, una diferente tinción basofílica permite la diferenciación de ambas zonas. No se observa la zona IV, ósea, por tratarse de fetos.

En el borde cubital del ligamento existe una configuración diferente, las fibras de colágeno
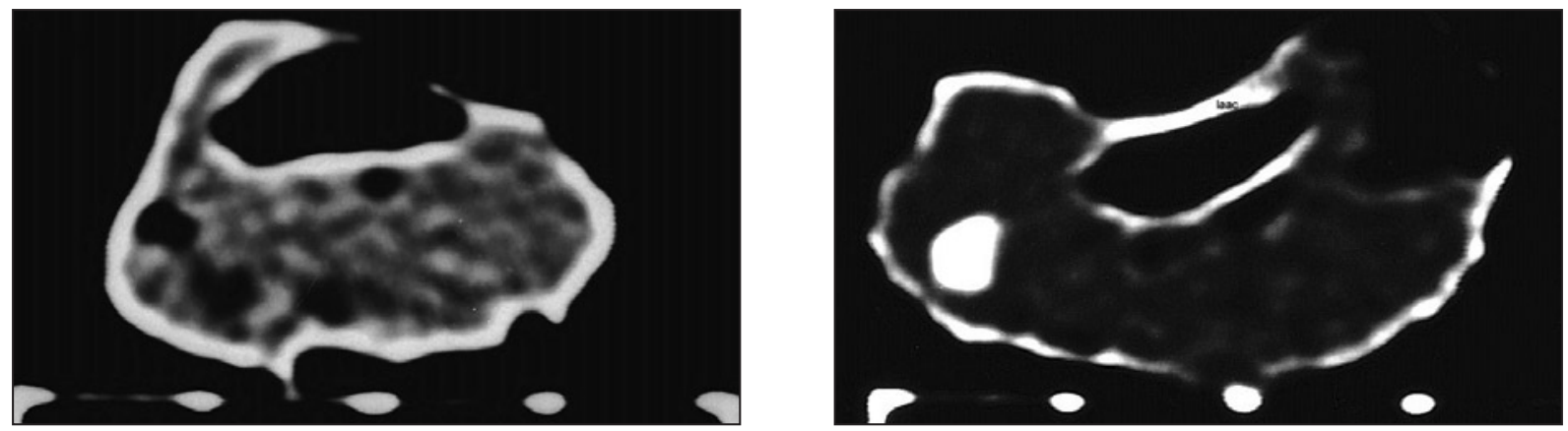

Figura 4. A: TAC. Túnel del carpo proximal. B: TAC. Túnel del carpo distal. 


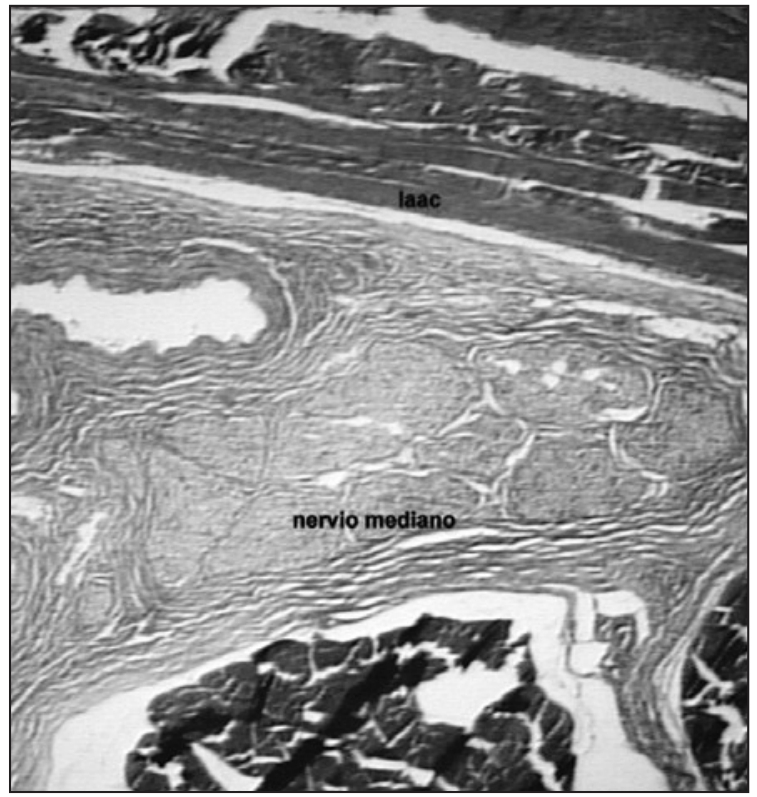

Figura 6. Relación de contigüidad entre el ligamento anular del carpo y el nervio mediano en el túnel del carpo.

divergen previo a su inserción, en dos grupos, uno superficial al paquete vascular y nervioso cubital, y otro profundo al mismo, que va insertarse a través de zonas transicionales de fibrocartílago y cartílago similares a las descritas en la inserción radial y que constituirán la pared cubital del túnel del carpo y la pared radial del canal de Guyon.

\section{DISCUSIÓN}

La dirección de la mayor parte de las fibras de colágeno del ligamento anular del carpo en su aspecto por microscopía óptica, se corresponde con el eje longitudinal del ligamento anular, que tiene una dirección transversal en la mano. No se objetivaron diferencias entre la parte proximal y la zona media del túnel del carpo, como describe Cobb et al. ${ }^{6}$.

El estudio de tomografía axial computerizada corrobora los estudios realizados en adultos ${ }^{8}$, permitiendo evaluar la morfología del túnel del carpo y del ligamento anular, diferenciando la fascia antebraquial del ligamento anular por localización anatómica, densidad radiológica y grosor.

El ligamento anular anterior del carpo presenta en su zona de inserción radial un esquema

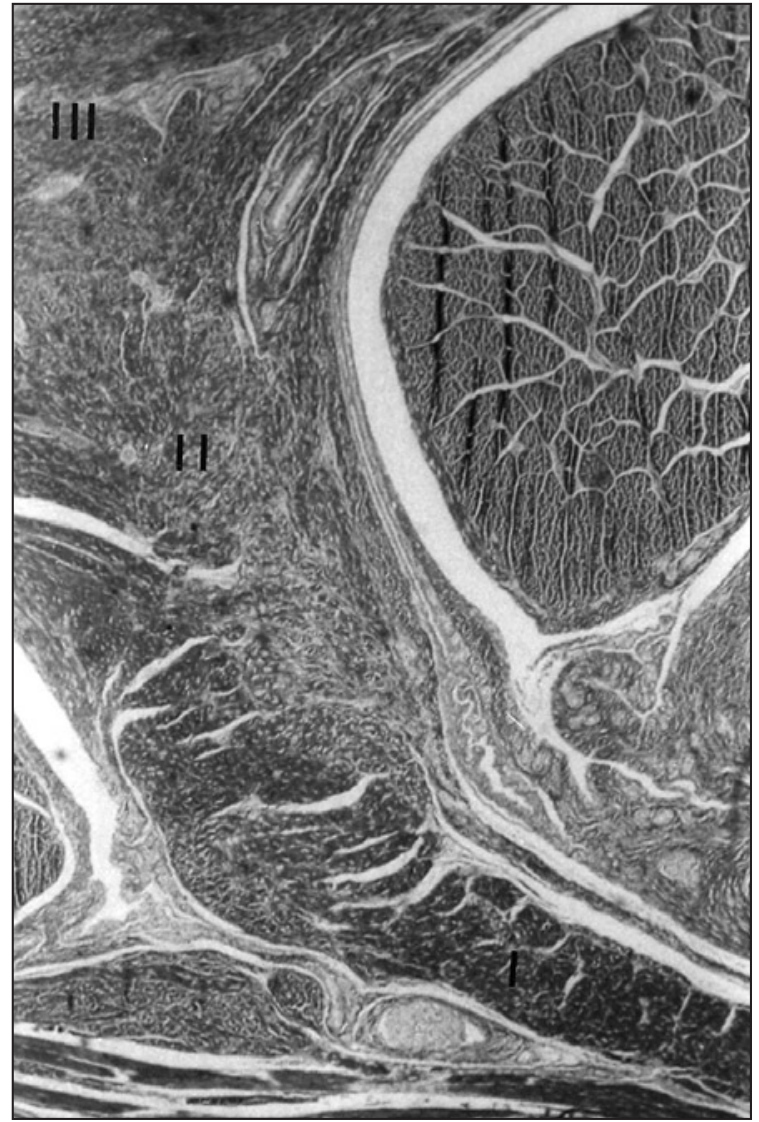

Figura 7. Inserción radial del ligamento anular del carpo, con sus tres zonas.

estructural similar al descrito en el tendón rotuliano y en el ligamento colateral interno de la rodilla en perros ${ }^{9,10}$. Existe una transición gradual, a través de capas desde una zona de ligamento con fibras de colágeno paralelas, a fibrocartílago y cartílago, no observándose la zona ósea ya que la osificación de los huesos del carpo no tiene lugar hasta después del nacimiento. Esta disposición en la zona de inserción cumpliría una función biomecánica, disminuyendo la sobrecarga de las tensiones a las cuales está sometido el ligamento ${ }^{11}$, similar a la de un ligamento estabilizador articular.

El punto de inserción de los ligamentos es más amplio que el ligamento mismo, relación que en el ligamento anular del carpo es aún mayor, lo que no obstante no modifica su esquema de inserción. Es decir, que las deformaciones de tensión a las que va estar sometido el ligamento anular hacen que se precise una amplia zona de transición ligamento-hueso, que transmita estas fuerzas. 
El concepto de relación por contigüidad, y no de continuidad, entre el ligamento anular del carpo y las capas más superficiales del epineuro del nervio mediano y con la sinovial de los flexores es importante, por cuanto significa que en la apertura quirúrgica del túnel del carpo, es posible seccionar el ligamento anular del carpo sin seccionar la capa continua de sinovial, protegiendo así el contenido del túnel, y seccionarla posteriormente en su borde cubital.

\section{BIBLIOGRAFÍA}

1. Fontaine C. Anatomie et biomécanique des canaux ostéofibreux palmaires du poignet. Cahier d'Enseignement de la Societé Française de Chirurgie de la Main. Paris: Expansion Scientifique, 1995: 77-91.

2. Denman EE. The volar carpal ligament. The Hand, 1979; 11: 22-7.

3. Landsmeer JMF. Atlas de Anatomía de la Mano. Barcelona: Editorial JIMS, 1979; 11-32.

4. Kauer JMG, Landsmeer JMF. Le poignet. Structure de la region carpienne. En: Tubiana R (Ed). Traité de Chirurgie de la Main. Paris: Masson, 1980; 176-91.
5. Spinner M. Kaplan's Functional and Surgical Anatomy of the Hand. 3nd Ed. Philadelphia: JB Lippincot Company, 1984; 261-3.

6. Cobb TK, Dalley BK, Poesteraro RH, Lewis RC. Anatomy of the flexor retinaculum. J Hand Sur Am, 1993; 18: 91-9.

7. McGrouther DA. The microanatomy of Dupuytren's disease. The Hand, 1982; 14: 215-36.

8. Zucker-Pinchoff B, Hermann G, Srinivasan R. Computed tomography of the carpal tunnel: A radioanatomical study. J Comp Ass Tom, 1981; 5: 525-28.
9. Cooper RR, Misol S. Tendon and ligament insertion. $J$ Bone Joint Surg Am, 1970; 52: 1-20.

10. Frank C, Amiel D, Woo SLY, Akeson W. Normal ligaments properties and ligament healing. Clin Orthop, 1985; 196: 15-25.

11. Schiavone Panni A, Franzese S, Denti M, Fabbriaciani C. Morphological evaluation of boneligament interaction in biological and artificial reconstruction. En: L'Hocine Yahia (Ed.). Ligaments and ligamentoplasties. Berlin: Springer-Verlag, 1997; 85-97. 